\title{
THE $L_{p}$ CONVERGENCE OF FOURIER-BESSEL \\ SERIES FOR $0<p<1$
}

\section{CHARLES STANDISH ${ }^{1}$}

The object of this note is to extend to Fourier-Bessel series a theorem of M. Riesz on $L_{p}$ convergence of trigonometric series. We prove the following

Theorem. If $f(x) \in L(0,1)$ then

$$
\lim _{N \rightarrow \infty} \int_{0}^{1}\left|f(x)-S_{N}(x)\right|^{p} d x=0, \quad 0<p<1,
$$

where $\nu \geqq-1 / 2$,

$$
\begin{aligned}
S_{N}(x) & =\sum_{1}^{N} b_{n}(2 x)^{1 / 2} J_{\nu}\left(u_{n} x\right) / J_{\nu+1}\left(u_{n}\right), \\
J_{\nu+1}\left(u_{n}\right) b_{n} & =2^{1 / 2} \int_{0}^{1} t^{1 / 2} J_{\nu}\left(u_{n} t\right) f(t) d t,
\end{aligned}
$$

and $\left\{u_{n}\right\}$ is the sequence of positive roots of $J_{\nu}(x)$.

In the proof of the theorem we need the

Lemma. If $f(x) \in L(a, b)$ then

$$
\hat{f}(x)=\int_{a}^{b} f(t)(x-t)^{-1} d t
$$

exists, is in $L_{p}(0<p<1)$ and

$$
\int_{a}^{b}|\hat{f}(x)|^{p} d x \leqq A\left(\int_{a}^{b}|f(x)| d x\right)^{p}
$$

where $A$ is independent of $f(x)$.

This lemma is an easy consequence of a result of Loomis ${ }^{2}[1, \mathrm{pp}$. 1085-1086].

We now proceed to the proof of the theorem. By the work of Wing [2, pp. 794-795],

Received by the editors July 29, 1956 and, in revised form, September 27, 1956.

1 This research was supported by the United States Air Force under Contract No. AF18(600)-685 monitored by the Office of Scientific Research.

2 This was pointed out to me by Professor Harry Pollard. 


$$
\begin{aligned}
\left|S_{N}(x)\right| \leqq & \int_{0}^{1}|f(t)| /(x+t) d t+\int_{0}^{1}|f(t)| /(2-x-t) d t \\
& +\frac{1}{2}\left|\int_{0}^{1}\left(A_{N} x\right)^{1 / 2} J_{\nu}\left(A_{N} x\right)\left(A_{N} t\right)^{1 / 2} J_{\nu+1}\left(A_{N} t\right) f(t) /(x-t) d t\right| \\
& +\frac{1}{2}\left|\int_{0}^{1}\left(A_{N} x\right)^{1 / 2} J_{v+1}\left(A_{N} x\right)\left(A_{N} t\right)^{1 / 2} J_{\nu}\left(A_{N} t\right) f(t) /(x-t) d t\right|
\end{aligned}
$$

where $A_{N}=(N+\nu / 2+1 / 4) \pi$. Since $\left(A_{N} x\right)^{1 / 2} J_{\nu}\left(A_{N} x\right)$ is bounded in $N$ and $x$ and $\left(A_{N} t\right)^{1 / 2} J_{\nu+1}\left(A_{N} t\right)$ is bounded in $N$ and $t[2, \mathrm{p} .796]$, by using the inequality

$$
\int|f(x)+g(x)|^{p} d x \leqq \int|f(x)|^{p} d x+\int|g(x)|^{p} d x \quad(0<p<1)
$$

we obtain

$$
\int_{0}^{1}\left|S_{N}(x)\right|^{p} d x \leqq(\text { constant })\left(\int_{0}^{1}|f(x)| d x\right)^{p} .
$$

By classical results on the convergence of Fourier-Bessel series $[4$, pp. 593-594, 612] given $\epsilon>0$ we can obtain a linear combination of functions $\left\{(2 x)^{1 / 2} J_{\nu}\left(u_{k} x\right)\right\}$ such that

$$
\int_{0}^{1}\left|f(x)-\sum_{1}^{N} c_{k_{\epsilon}}(2 x)^{1 / 2} J_{\nu}\left(u_{k} x\right)\right| d x<\epsilon
$$

and

$$
\int_{0}^{1}\left|f(x)-\sum_{1}^{N} c_{k_{\epsilon}}(2 x)^{1 / 2} J_{\nu}\left(u_{k} x\right)\right|^{p} d x<\epsilon .
$$

The remainder of the proof proceeds as in the case of ordinary Fourier series [3, p. 153].

Write $f(x)$ in the form $f_{1}(x)+f_{2}(x)$ where

$$
f_{1}(x)=\sum_{1}^{N} c_{k_{\epsilon}}(2 x)^{1 / 2} J_{\nu}\left(u_{k} x\right)
$$

and $S_{N}(x)$ in the form

$$
S_{N}(x)=S_{N_{1}}(x)+S_{N_{2}}(x)
$$

where $S_{N_{1}}(x)$ and $S_{N_{2}}(x)$ are the $N$ th partial sums of the FourierBessel series of $f_{1}(x)$ and $f_{2}(x)$ respectively. Then 


$$
\begin{aligned}
& \int_{0}^{1}\left|f(x)-S_{N}(x)\right|^{p} d x \\
& \quad=\int_{0}^{1}\left|f_{1}(x)-S_{N_{1}}(x)+f_{2}(x)-S_{N_{2}}(x)\right|^{p} d x \\
& \leqq \int_{0}^{1}\left|f_{1}(x)-S_{N_{1}}(x)\right|^{p} d x+\int_{0}^{1}\left|f_{2}(x)-S_{N_{2}}(x)\right|^{p} d x
\end{aligned}
$$

but, if $N$ is large enough, $S_{N_{1}}(x)=f_{1}(x)$ since $f_{1}(x)$ is merely a finite linear combination of functions from the set $\left\{(2 x)^{1 / 2} J_{\nu}\left(u_{k} x\right)\right\}$. Finally

$$
\begin{aligned}
\int_{0}^{1}\left|f(x)-S_{N}(x)\right|^{p} d x & \leqq \int_{0}^{1}\left|f_{2}(x)\right|^{p} d x+\int_{0}^{1}\left|S_{N_{2}}(x)\right|^{p} d x \\
& \leqq \epsilon+A \int_{0}^{1}\left|f_{2}(x)\right|^{p} d x=(A+1) \epsilon
\end{aligned}
$$

\section{REFERENCES}

1. L. H. Loomis, A note on the Hilbert transform, Bull. Amer. Math. Soc. vol. 52 (1946) pp. 1082-1086.

2. G. Wing, The mean convergence of orthogonal series, Amer. J. Math. vol. 72 (1950) pp. 792-808.

3. A. Zygmund, Trigonometrical series, Warsaw, 1935.

4. G. N. Watson, A treatise on the theory of Bessel functions, Cambridge, 1944. CORNell University 